\title{
Surgeries for the Heart and Abdominal Aorta in a Patient with Heparin-Induced Thrombocytopenia: Manifestations Following Initial Heart Surgery
}

\author{
Shoh Tatebe ${ }^{1}$, Makoto Taoka ${ }^{2}$, Imun Tei ${ }^{2}$, Shuko Nakamura ${ }^{2}$, Ei-ichi Tei ${ }^{2}$ \\ ${ }^{1}$ Department of Thoracic and Cardiovascular Surgery, Mito Saiseikai General Hospital, Mito City, Japan; ${ }^{2}$ Department of Cardio- \\ vascular Surgery, Ayase Heart Hospital, Tokyo, Japan. \\ Email: statebe@yahoo.com
}

Received December $4^{\text {th }}, 2011$; revised January $24^{\text {th }}, 2012$; accepted February $6^{\text {th }}, 2012$

\begin{abstract}
A 72-year-old man with abdominal aortic aneurysm and angina pectoris underwent coronary artery bypass grafting (CABG) prior to abdominal aortic surgery. Perioperatively, he developed thrombocytopenia $\left(29,000 \mathrm{~mm}^{3}\right)$, which was suggested as heparin-induced thrombocytopenia. Cardiac status was also aggravated at the same time, suggestive of bypass graft thrombosis. The results of platelet factor 4 (pf4) antibody test were negative, but platelet aggregation test was positive for heparin. Heparin was immediately discontinued, and replaced by argatroban; the patient's cardiac status improved. One month later, he underwent abdominal aortic surgery using argatroban without issues related to anticoagulation and hemostasis. Autologous donated fresh blood prepared by the "switch-back method" was also used in this case, and its value was confirmed.
\end{abstract}

Keywords: Heparin-Induced Thrombocytopenia

\section{Introduction}

Heparin-induced thrombocytopenia (HIT) is an immunemediated clotting disorder caused by frequent and extensive heparin exposure. The clinical manifestations of HIT are thrombocytopenia and thrombosis, usually with devastating consequences, such as limb ischemia, stroke, pulmonary embolism, or death [1]. The clinical picture of HIT after cardiac surgery is complicated, and may result in fetal thrombotic events, such as myocardial infarction. Here, we report a case of HIT revealed after cardiac surgery, which was treated successfully without sequelae, and the patient subsequently underwent abdominal aortic surgery without HIT-related adverse events.

\section{Case Presentation}

A 72-year-old man was referred by a local general hospital for surgical treatment of an abdominal aortic aneurysm (AAA). His medical history included myocardial infarction on posterior and lateral walls over 20 years previously, for which conservative treatment was performed because catheter intervention for the coronary artery was not available. He was diagnosed as diabetic 1 month prior to presentation at a local general hospital, where he had been hospitalized. Treatment for diabetes was initiated and systemic workup was performed. An
AAA $5 \mathrm{~cm}$ in diameter was found during systemic workup, but he was asymptomatic. However, coronary angiography (CAG) revealed multiple stenoses on the coronary arteries, including $75 \%$ stenosis of the left main trunk. Due to concerns regarding life-threatening perioperative complications, he was scheduled for coronary artery bypass grafting (CABG) prior to surgery for AAA.

The patient underwent CABG (three vessels) with cardiopulmonary bypass (CPB). An intraaortic balloon pumping (IABP) catheter was introduced after induction of general anesthesia to avoid critical intraoperative myocardial infarction. The initial postoperative course was uneventful, and IABP was discontinued on postoperative day 1 (POD1). However, platelet count decreased suddenly to $29,000 / \mathrm{mm}^{3}$ on POD2 and $30,000 / \mathrm{mm}^{3}$ on POD3. Although platelet factor 4 (pf4) antibody test was negative, heparin was discontinued immediately and replaced with argatroban due to suspected heparin-induced thrombocytopenia (HIT). Cardiac status became aggravated, and intravenous argatroban was started at $20 \mathrm{mg} /$ day due to concerns regarding graft occlusion. Platelet counts normalized and heart failure improved. Postoperative CAG indicated that all grafts were tolerated and patent. Platelet aggregation test (PAT) was positive at $10 \mathrm{mi}-$ cromol heparin, confirming diagnosis of HIT type I.

The patient underwent abdominal aortic surgery one 
month after CABG. Preoperatively, 6 units of autologous blood were obtained using erythropoietin. In the operating room, 6 units of autologous fresh blood were taken by the "switch-back method". All pressure lines and the circuit of the autologous blood transfusion device were replaced with saline containing argatroban or acid-citrate-dextrose solution. Prior to clamping the arteries, 8.5 $\mathrm{mg}(0.1 \mathrm{mg} / \mathrm{kg})$ of argatroban was administered as a bolus, and maintained at $10-15 \mathrm{mg} / \mathrm{h}$ to achieve activated coagulation time (ACT) $>200$ s. The AAA was successfully replaced by a vascular prosthesis. Argatroban was discontinued after reperfusion, and autologous fresh blood was given. No bleeding was noted, but as there is no antidote to argatroban, it was necessary to wait for over 90 min until ACT had normalized. Postoperatively, argatroban was resumed and then switched to oral warfarin. The postoperative course was uneventful and the patient was discharged on POD14. PAT were repeated and remained positive with heparin for 3 years.

\section{Discussion}

In the present case with postoperative manifestations of HIT after CABG, the patient underwent abdominal aortic surgery using argatroban instead of heparin. This case raised the issue of the time between onset of HIT and treatment. Perioperatively, platelet count may be lower than $100,000 / \mathrm{mm}^{3}$ after cardiac surgery. Therefore, it is quite difficult to make a definite diagnosis in patients with initial manifestations of HIT. IABP may have been responsible for thrombocytopenia in this case. Therefore, another day was required for suspicion of HIT, and the initiation of treatment was delayed. Most patients undergoing cardiovascular surgery have been exposed to heparin during treatment or examination by the cardiology service. Postoperative manifestations of HIT tend to occur in patients referred from institutes other than cardiology services or requiring emergent surgery. In the 3 years since treating this patient, three other patients have presented with HIT postoperatively in our department; two of these patients underwent emergent surgery due to acute mitral regurgitation and dissecting aortic aneurysm. Based on these cases, we have implemented preoperative PAT using heparin in patients without heparin exposure. The pf4 antibody test is a simple means of identifying HIT type II, but does not provide information to exclude a diagnosis of HIT type I. Even by ELISA, the presence of pf 4 antibodies can be confirmed only with a delay of 2 days or more in approximately $50 \%$ of patients in whom HIT develops [2]. At present, PAT using heparin is much faster even in cases with HIT type I in our institution, but no HIT patients have been encountered to date.

Another issue is the initial treatment for HIT. The current recommendation is to discontinue heparin in cases of suspected HIT and begin treatment with direct thrombin inhibitors, such as hirudin/bivalrudin, danaparoid, and argatroban [3]. Danaparoid and argatroban are available in Japan. Argatroban is a derivative of l-arginine, which binds reversibly to the active site of thrombin, and is approved for treating HIT by the FDA as well as recombinant hirudin (lepirudin). We chose argatroban because it has a number of advantages, including specific rapid onset, and is safe in patients with renal impairment. Perioperatively, when HIT was suspected, hemodynamic status was aggravated in this case. As coronary angiography was not performed, there was no actual evidence of graft occlusion. However, it was suspected that bypass would have a risk of thrombotic occlusion. In this patient, cardiac status settled down after initiation of argatroban treatment, suggesting thrombolysis in the native coronary arteries and/or bypass grafts. Argatroban would be effective for HIT not only in this case, but also in the three HIT patients we encountered previously.

The other issue is the treatment strategy for the AAA. Over the past decade methods for endovascular repair (EVAR) of aortic aneurysms have been developed, and the indications have been expanded from the descending aorta to other branched aortae, such as the aortic arch, thoracoabdominal aorta, and abdominal aorta. At the time of surgery for AAA in the present case, approval for EVAR in Japan was strictly limited to elderly patients or those with difficulties in conventional surgical repair, such as previous abdominal surgery. Therefore, this patient underwent conventional surgical repair of AAA with argatroban treatment. There have been no previous reports of EVAR in patients known to have HIT, but two cases of HIT manifestations following EVAR by graft occlusion were reported $[4,5]$. There have been a small number of reports and reviews regarding EVAR in patients with preoperatively diagnosed HIT, but many reviews regarding cardiovascular surgery the principles of which can be summarized as: 1) wait until pf4 antibody level subsides, then use heparin; 2) use alternative anticoagulation, such as danaproid or hirudin/bivalrudin to replace heparin. Warkentin and Greinacher included use of heparin with epoprostenol/tirofiban as an option [1]. As mentioned above, not all cases of HIT are diagnosed by pf 4 antibody, and therefore there are HIT type II patients negative for pf 4 antibody. We encountered a patient on hemodialysis who underwent surgery to create an internal shunt when he was found to have HIT. Although pf4 antibody was negative, he developed severe thrombosis with a trace amount of heparin. In this case, although pf4 antibody was negative, PAT remained positive for heparin for at least 3 years. The PAT results suggested high risk in this case, and we were hesitant to use heparin. We again chose argatroban bolus and con- 
tinuous infusion on abdominal aortic surgery. There were no difficulties in monitoring clotting activities by ACT or changing the dose accordingly. Questions have been raised regarding whether argatroban is well tolerated in CPB during cardiac surgery [1,3]. However, argatroban was effective for vascular surgery not only in our patient but also in many previously reported cases [6,7]. There is no antidote for argatroban, such as protamine for heparin. In this case, after reperfusion, it was necessary to wait more than 90 min until ACT normalized. During this time, autologous fresh blood was returned and no bleeding issues occurred. The so-called "switch-back method" involves transfusion of homologous/autologous blood before injection of anticoagulant, storage of fresh blood, and return after CPB or reperfusion. This method has been recognized as effective in normalizing clotting activities after СРВ in cardiac surgery. We also use this method in cases of vascular disease and it was beneficial in HIT.

\section{REFERENCES}

[1] T. E. Warkentin and A. Greinacher, "Heparin-Induced Thrombocytopenia and Cardiac Surgery,” Annals of Thoracic Surgery, Vol. 76, No. 6, 2003, pp. 2121-2131. doi:10.1016/j.athoracsur.2003.09.034

[2] H. Gulbins, T. Chavez and J. Ennker, "Postoperative Manifestation of Heparin-Induced Thrombocytopenia with Intracavitary Thrombosis: Diagnostic Pitfalls and Conservative Therapy," Jounal of Thoracic and Cardiovas- cular Surgery, Vol. 133, No. 3, 2007, pp. 809-810. doi:10.1016/j.jtcvs.2006.11.004

[3] G. S. Murphy and J. H. Marymont, "Alternative Anticoagulation Management Strategies for the Patient with Heparin-Induced Thrombocytopenia Undergoing Cardiac Surgery,” Journal of Cardiothoracic and Vascular Anesthesia, Vol. 21, No. 1, 2007, pp. 113-126. doi:10.1053/j.jvca.2006.08.011

[4] T. A. M. Chuter, L. K. Pak, R. L. Gordon, L. M. Reilly and L. M. Messina, "Heparin-Induced Thrombocytepenia and Graft Thrombosis Following Endovascular Aneurysm Repair," Journal of Endovascular Therapy, Vol. 10, No. 6, 2003, pp. 1087-1090. doi:10.1583/1545-1550(2003)010<1087:HTAGTF $>2.0 . C$ $\underline{\mathrm{O} ; 2}$

[5] R. Kolluri, K. Rocha-Singh, T. Sarac and J. R. Bartholomew, "Heparin-Induced Thrombocytopenia with Thrombosis after Endovascular Anerysm Repair," Vascular and Endovascular Surgery, Vol. 43, No. 1, 2009, pp. 89-92. doi:10.1177/1538574408322660

[6] J. T. Edwards, J. K. Hamby and N. K. Worrall, "Successful Use of Argatroban as a Heparin Substitute during Cardiopulmonary Bypass: Heparin-Induced Thrombocytopenia in a High-Risk Cardiac Surgical Patient," Annals of Thoracic Surgery, Vol. 75, No. 5, 2003, pp. 1622-1624. doi:10.1016/S0003-4975(02)04782-3

[7] S. E. Hallman, L. Hebbar, J. Robison and W. E. Uber, "The Use of Argatoroban for Carotid Endarterectomy in Heparin-Induced Thrombocytopenia," Anesthesia and Analgesia, Vol. 100, No. 4, 2005, pp. 946-948. doi:10.1213/01.ANE.0000146940.47989.5F 